\title{
Voluntary Running Enhances Hippocampal Proliferation by Increasing Hippocampal NGF, BDNF, and IGF-1
}

\author{
Bo Xu ${ }^{1,2}$, Xianliang Zhang ${ }^{3}$, Chenghui Song ${ }^{4}$, Fei Liang ${ }^{2}$, Li Zhang ${ }^{5}$ \\ ${ }^{1}$ Key Laboratory of Adolescent Health Assessment and Exercise Intervention of Ministry of Education, East China Normal University, \\ Shanghai, China \\ ${ }^{2}$ School of Physical Education \& Health Care, East China Normal University, Shanghai, China \\ ${ }^{3}$ College of Physical Education, Shandong University, Jinan, China \\ ${ }^{4}$ Department of Neuroscience, the Scripps Research Institute, Jupiter, Florida, USA \\ ${ }^{5}$ Division of Physical Education, China University of Mining \& Technology, Beijing, China
}

\section{Email address:}

bxu@tyxx.ecnu.edu.cn (Bo Xu)

\section{To cite this article:}

Bo Xu, Xianliang Zhang, Chenghui Song, Fei Liang, Li Zhang. Voluntary Running Enhances Hippocampal Proliferation by Increasing Hippocampal NGF, BDNF, and IGF-1. Advances in Biochemistry. Vol. 5, No. 1, 2017, pp. 1-6. doi: 10.11648/j.ab.20170501.11

Received: January 4, 2017; Accepted: January 25, 2017; Published: March 4, 2017

\begin{abstract}
Neurotrophic factors and growth factors are known to have positive effects on hippocampal proliferation. However, previous findings on the effects of these factors on exercise-induced hippocampal proliferation are inconsistent. In this study, we investigated the effects of voluntary running on hippocampal proliferation and the expression levels of nerve growth factor (NGF), brain-derived neurotrophic factor (BDNF), and insulin-like growth factor 1 (IGF-1) in hippocampus. Twenty-four male C57BL/6 mice were randomly divided into four groups: control groups 1 and $2(\mathrm{C} 1, \mathrm{n}=6 ; \mathrm{C} 2, \mathrm{n}=6)$ and running groups 1 and $2(\mathrm{R} 1, \mathrm{n}=6 ; \mathrm{R} 2, \mathrm{n}=6)$. Mice from $\mathrm{C} 1$ and $\mathrm{C} 2$ were placed individually in standard cages for eight weeks; mice from R1 and R2 were housed individually in cages with a running wheel for eight weeks. At the fifth week, C1 and R1 mice were injected with BrdU for 7 consecutive days. On the first day of the ninth week, the $\mathrm{C} 1$ and R1 mice were anaesthetized and hippocampal neuronal proliferation was assessed using immunohistochemistry; the mice in C2 and R2 were sacrificed to test the expression levels of NGF, BDNF, and IGF-1 mRNA in hippocampus using RT-PCR. The results indicated that voluntary running significantly increased the number of BrdU-positive cells in the dentate gyrus (DG), and significantly upregulated the levels of NGF, BDNF, and IGF-1 mRNA in the hippocampus. In conclusion, our findings indicated that hippocampal proliferation was enhanced by voluntary running, which was associated with increased NGF, BDNF, and IGF-1 mRNA levels in the hippocampus.
\end{abstract}

Keywords: Voluntary Exercise, Hippocampal Proliferation, NGF, BDNF, IGF-1

\section{Introduction}

Many studies have confirmed that neurogenesis continues in discrete regions of the adult brain, especially in the subgranular zone (SGZ) of the dentate gyrus (DG) and the subventricular zone (SVZ) of the lateral ventricles [1, 2]. During adult hippocampal neurogenesis, newly generated cells can proliferate, differentiate, mature, and integrate into functional neural networks [3]. Aging or dead cells are substituted by new cells. Neuronal proliferation plays a vital role in the maintenance of hippocampal structure and function. Some neurotrophic factors or growth factors have been demonstrated to be involved in mediating proliferation in the hippocampus. For instance, Birch et al [4] showed that chronic intracerebroventricular infusion of nerve growth factor (NGF) promoted neuronal proliferation in the hippocampal DG. In contrast, conditional knockout brain-derived neurotrophic factor (BDNF) resulted in the reduction of hippocampal proliferation [5]. In addition, Aberg et al [6] found that insulin-like growth factor 1 (IGF-1) had a direct proliferative effect in adult hippocampal progenitor cells.

Some studies also examined the effects of BDNF on exercise-induced hippocampal neurogenesis. For instance, 
treadmill exercise increased hippocampal BDNF expression, and promoted hippocampal proliferation [7-9]. However, another study reported that treadmill exercise increased hippocampal proliferation but did not increased BDNF protein [10]. In addition, Marlatt et al [11] divided 9-month-old mice into control group, 6-month running group and 1-month running group. Exercise group was housed with a running wheel. All animals were injected with BrdU in the first 10 days. The results showed that 6-month voluntary wheel running increased hippocampal proliferation and BDNF protein, whereas 1-month voluntary wheel running did not enhance hippocampal proliferation and BDNF protein. The reason may be correlated with the duration of voluntary running. Therefore, in the current study we examined the effects of 8-week voluntary wheel running on hippocampal proliferation and hippocampal BDNF mRNA level.

In a previous study, mice were divided into three groups: control mice (C), restraint-stressed mice (RS), and voluntary exercise + restraint-stressed mice $(\mathrm{VE}+\mathrm{RS})$. The results showed that the mice in $\mathrm{VE}+\mathrm{RS}$ group had more BrdU+ cells in the hippocampus and IGF-1 protein in the cerebral cortex than mice of RS group. These indicated that voluntary exercise improved hippocampal proliferation and IGF-1 level in the cerebral cortex of stressed mice [12]. In another study, exercise-induced hippocampal proliferation and IGF-1 protein level in the brain was decreased, if the entrance of circulating IGF-1 into brain was blocked by subcutaneous infusion of a blocking IGF-1 antiserum to rats undergoing exercise training [13].

In addition, wheel running could increase hippocampal NGF level [14], whereas no previous study has examined the effect of exercise-induced hippocampal NGF on hippocampal proliferation. Therefore, the purpose of the present study was to investigate whether or not hippocampal proliferation was enhanced in response to eight weeks of voluntary wheel running in mice. We further investigated the expression levels of NGF, BDNF, and IGF-1 mRNA in the hippocampus.

\section{Materials and Methods}

\subsection{Animals}

A total of 24 male C57B1/6 mice (5 weeks of age; SLAC Laboratory Animal Company, Shanghai, China) were used in this study. The mice were randomly divided into four groups: control groups 1 and $2(\mathrm{C} 1, \mathrm{n}=6 ; \mathrm{C} 2, \mathrm{n}=6)$ and running groups 1 and $2(\mathrm{R} 1, \mathrm{n}=6 ; \mathrm{R} 2, \mathrm{n}=6)$. The mice were housed individually in temperature-controlled conditions at $23^{\circ} \mathrm{C}$ with a 12-h light/dark cycle. All mice had access to food and water ad libitum. All experimental procedures were approved by the Experimental Animal Care and Use Committee at East China Normal University [license number for the use of laboratory animals: SYXK (Shanghai) 2004-0001] and conformed to the guidelines for the use of laboratory animals published by the People's Republic of China Ministry of Health (25 January 1998).

\subsection{Exercise Protocol and Injection of BrdU}

R1 and R2 mice were exposed to voluntary wheel running (wheel diameter, $10 \mathrm{~cm}$ ) for 8 weeks. The number of revolutions of the wheel was monitored by a counter and the running distance was determined by multiplying the number of revolutions by wheel circumference [15]. C1 and C2 mice were housed under standard conditions for 8 weeks. At week five, newly generated cells in the DG of the R1 and C1 mice were date-labeled with $50 \mathrm{mg} / \mathrm{kg} \mathrm{BrdU}$ (intraperitoneal injection after the mice were weighed at 5 PM; Sigma, Hamburg, Germany) for 7 consecutive days. BrdU is a thymine nucleoside analogue that replaces thymidine, and can be incorporated into the newly synthesized DNA of replicating cells (during the $\mathrm{S}$ phase of the cell cycle).

\subsection{Tissue Preparation}

On the first day of week 9, BrdU-treated mice (R1 and C1) were anaesthetized with $1 \%$ sodium pentobarbital and transcardially perfused with $0.9 \% \mathrm{NaCl}$ solution followed by $4 \%$ paraformaldehyde. The brains were stored in $4 \%$ paraformaldehyde overnight at $4^{\circ} \mathrm{C}$. After fixation, all brains were transferred to $15 \%$ sucrose until the brains sank to the bottom, and then transferred to $30 \%$ sucrose for 30 hours. After all brains sank to the bottom, they were cut in the coronal plane (20- $\mu \mathrm{m}$ sections) with a dry ice-cooled copper block on a sliding micrcotome (Leica, Bensheim, Germany) and stored in $\mathrm{OCT}$ at $-80^{\circ} \mathrm{C}$ until BrdU staining. At the same time, R2 and C2 mice were sacrificed. The hippocampi were collected, frozen in liquid nitrogen immediately, and stored at $-80^{\circ} \mathrm{C}$ until use for gene expression analysis by RT-PCR.

\subsection{Immunohistochemistry}

For BrdU detection, sections were rinsed in PBS ( 5 min $\times$ 3 ), and incubated in $50 \%$ formamide for $2 \mathrm{~h}$ at $65^{\circ} \mathrm{C}$, and hydrolyzed in $2 \mathrm{~N} \mathrm{HCl}$ at $37^{\circ} \mathrm{C}$. The sections were then rinsed three times with $0.1 \mathrm{M}$ borate buffer, incubated in $3 \% \mathrm{H}_{2} \mathrm{O}_{2}$ and $0.3 \%$ Triton $\mathrm{X}-100$ for $30 \mathrm{~min}$ each at room temp, and blocked for $1 \mathrm{~h}$ with $3 \%$ BSA. The sections were then incubated with sheep anti-BrdU (1:200 dilution, ab1893, Abcam) in $2 \%$ BSA overnight at $4{ }^{\circ} \mathrm{C}$. On the second day, the sections were incubated with fluorescein isothiocyanate (FITC)-conjugated rabbit anti-sheep antibodies (1:500 dilution, 6150-02, Southern Biotech) in 2\% BSA for 1 hour at RT, kept in the dark, and then incubated in 4',6-Diamidino-2-phenylindole dihydrochloride (DAPI) for 2 hours. Finally, fluorescent signals were imaged under a fluorescence microscope (Leica AF6000, Germany).

\subsection{RT-PCR}

Total RNA was extracted from the hippocampus with Trizol reagent after homogenate. The purity of RNA was measured by an ultraviolet spectrometer. RNA was used for reverse transcription when OD260/OD280 $\geq 1.8$. cDNA was acquired using the ReverTra Ace qPCR RT kit (Toyobo Co. Ltd., Osaka, Japan). mRNA was measured by the Toyobo 
SYBR-green PCR kit (Toyobo Co. Ltd). mRNA abundance was normalized to that of $\beta$-actin, which was used as the internal control. The primer sequences are shown in Table 1.

Table 1. Primer Sequence.

\begin{tabular}{|c|c|}
\hline Genes & Primer Sequences \\
\hline NGF & $\begin{array}{l}\text { Forward primer: GAC AAC AGA TGA GAA GCA GGC } \\
\text { Reverse Primer: AGG AGA GTG TGG AGG GGG }\end{array}$ \\
\hline BDNF & $\begin{array}{l}\text { Forward primer: GTT CCA CCA GGT GAG AAG AGTG } \\
\text { Reverse Primer: AAA GTG TCA GCC AGT GAT GTCG }\end{array}$ \\
\hline IGF-1 & $\begin{array}{l}\text { Forward Primer: } \\
\text { Reverse Primer : }\end{array}$ \\
\hline$\beta$-actin & $\begin{array}{l}\text { Forward Primer: CTC TTT TCC AGC CTT CCT TCT } \\
\text { Reverse Primer: TGG AAG GTG GAC AGT GAGG }\end{array}$ \\
\hline
\end{tabular}

\subsection{Statistical Analysis}

All statistical analyses were performed using GraphPad Prism (version 5.0; GraphPad Software Inc., San Diego, California) and SPSS version 17.0. The t test was used to compare differences between the control and running groups. All data are presented as means \pm SEM. The level of significance was set at $\mathrm{P}<0.05$ or $\mathrm{P}<0.01$ (two-sided).

\section{Results}

\subsection{Daily Running Distance}

The daily average revolutions of mice from R1 and R2 over the eight-week period were $23625.70 \pm 7745.70$. The wheel circumference is $0.314 \mathrm{~m}$. Thus, the daily average running distance was $7418.47 \pm 2432.15 \mathrm{~m}$.

\subsection{Effect of Voluntary Running on the Number of BrdU+ Cells in the DG}

The phenotype of BrdU-positive cells was assessed by single immunofluorescence labeling for BrdU. Fluorescence microscopy was used to count the number of single-labeled BrdU-positive cells in the DG (Fig. 1 a, b). There was a significant difference in the number of BrdU-positive cells in the $\mathrm{R} 1$ and $\mathrm{C} 1$ groups $(\mathrm{C} 1: 71.83 \pm 15.52, \mathrm{R} 1: 257.83 \pm$ 56.74, $\mathrm{P}<0.01$; Fig. $1 \mathrm{c}$ ). This result suggested that voluntary running improved cell proliferation in the DG of the hippocampus.

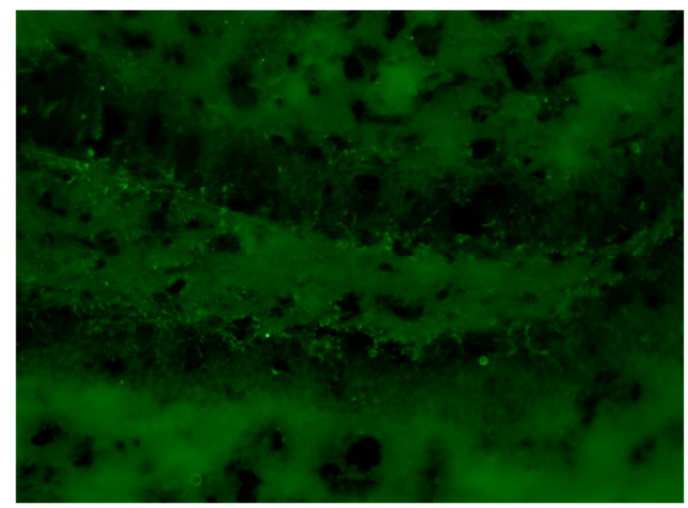

(a)

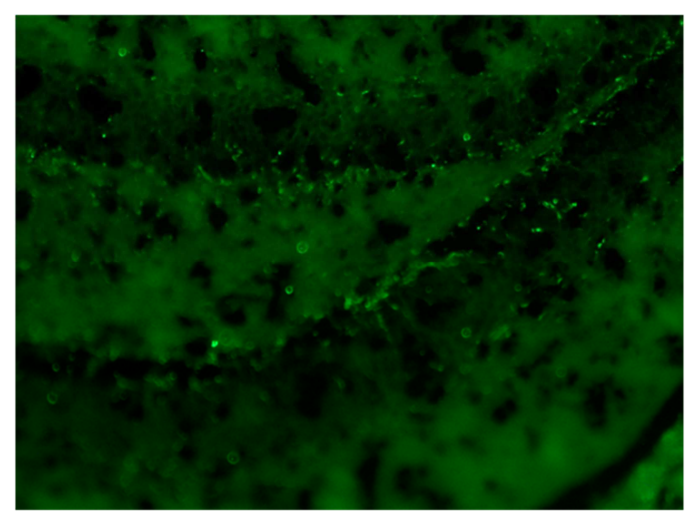

(b)

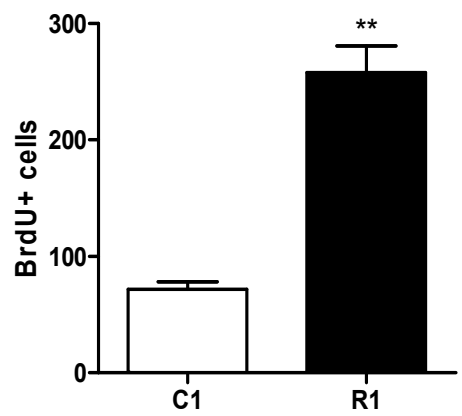

(c)

Fig. 1. Effect of voluntary running on the number of BrdU-positive cells in the $D G$. (a) A representative image showing BrdU+cells in the dentate gyrus $(D G)$ of the C1 group. (b) A representative image showing BrdU+cells in the DG of the Rl group. (c) Quantitative data of BrdU+cells in the two groups. All data are expressed as means $\pm S E M(n=6$ in each group). $* * P<0.01$ vs. $C 1$.

\subsection{Effect of Voluntary Running on the Expression of NGF, $B D N F$, and IGF-1 $m R N A$ in the Hippocampus}

There was a significant increase in the NGF mRNA (C2: $1.00 \pm 0.34, \mathrm{R} 2: 3.76 \pm 0.47, \mathrm{P}<0.01), \mathrm{BDNF}$ mRNA $(\mathrm{C} 2$ : $1.00 \pm 0.39, \mathrm{R} 2: 3.56 \pm 1.42, \mathrm{P}<0.01)$, and IGF-1 mRNA $(\mathrm{C} 2: 1.00 \pm 0.73, \mathrm{R} 2: 2.51 \pm 0.86, \mathrm{P}<0.01)$ in the hippocampus of the R2 group compared to that in the $\mathrm{C} 2$ group (Fig. 2). These results indicated that voluntary running increased the mRNA expression levels of NGF, BDNF, and IGF-1 in the hippocampus.

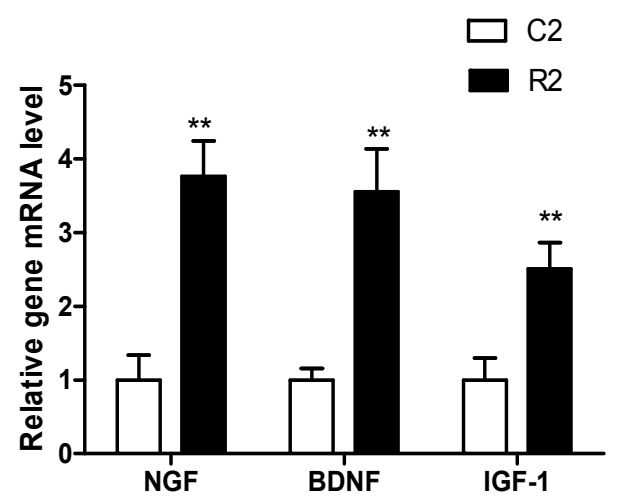

Fig. 2. Effect of voluntary running on the expression of $N G F, B D N F$, and $I G F-1 \mathrm{mRNA}$ in the hippocampus. The expression levels of $N G F, B D N F$, and $I G F-1$ between the $C 2$ and $R 2$ groups were determined by RT-PCR. Data are expressed as means $\pm S E M(n=6$ in each group). $* * P<0.01$ vs. $C 2$. 


\section{Discussion}

\subsection{Voluntary Running Increased Proliferation in the DG}

In the present study, we found that the daily running distance was approximately $7.4 \mathrm{~km}$, which was consistent with the results of a previous study of Allen, who found that the average daily running distance of C57BL/ 6 mice was 6.8 $\mathrm{km} \mathrm{[16].} \mathrm{We} \mathrm{examined} \mathrm{the} \mathrm{number} \mathrm{of} \mathrm{proliferative} \mathrm{neural}$ cells using BrdU immunofluorescence staining. There were significantly higher BrdU-positive cells in the DG of group $\mathrm{R} 1$ than in the DG of group $\mathrm{C} 1(\mathrm{P}<0.01$, Fig. 1), suggesting that hippocampal proliferation was increased in mice housed with running wheel. In van Praag's [17] study, adult mice were divided into learner, swimmer, runner, enriched, and control groups, which were given a daily intraperitioneal injection of BrdU. They found that only runner and enriched increased BrdU-positive cells in the DG. In subsequent research, they divided 3-month-old mice into sedentary and running groups. Runners had unlimited access to a running wheel for 45 days. The result revealed that wheel running increased the number of BrdU-positive cells in the DG [18]. These results indicated that voluntary wheel running could increase hippocampal proliferation, which was in accordance with our current results. Another study about treadmill exercise on hippocampal proliferation also showed that treadmill running for 5 weeks promoted neural stem cells (NSC) proliferation in the DG [8]. In another interesting study, rats were randomly divided into four groups: low-intensity, moderate-intensity, and high-intensity exercise groups and control group. The results showed that forced low- or moderate-intensity treadmill exercise for one week enhanced hippocampal neurogenesis in rats [19]. Therefore, low-intensity aerobic exercise, including voluntary exercise and forced exercise, could promote hippocampal proliferation. The positive effect of exercise on hippocampal proliferation may be associated with increased neurotrophic factors or growth factors.

\subsection{Voluntary Running Enhanced Proliferation by Increasing NGF, BDNF, and IGF-1}

Neurotrophic factors such as NGF and BDNF, are extracellular proteins that play vital roles in neurogenesis. NGF was the first neurotrophic factor to be discovered. It is found in the neurons of the hippocampus, olfactory bulb, and cerebral cortex. In the present study, we found that the NGF mRNA level in the hippocampus was increased following voluntary wheel running for 8 weeks. In previous studies, Neeper et al [14] found that voluntary running significantly increased NGF mRNA in the hippocampus, and O'Callaghan et al [20] demonstrated that long-term exercise prevented or reversed age-related decline in the expression of NGF mRNA. These results are consistent with our current results. In addition, we also found that there was an increase in NGF accompanied by hippocampal proliferation, indicating that hippocampal NGF has a positive impact on hippocampal proliferation following voluntary exercise. Regarding subcellular mechanism, Kim et al [21] found that NGF activated TrkA receptor, phosphorylated Shc, activated Ras phosphorylation, and induced ERK activity, which in turn phosphorylated CREB to promote proliferation. Therefore, the mechanism may be that exercise increases NGF, which promotes proliferation in the DG through MAPK pathway.

Chronic BDNF injection into the adult DG resulted in increased neurogenesis of granule cells [22], whereas conditional knockdown of BDNF has been shown to reduce the proliferation in the adult DG in vivo [5]. Another study found that 3 weeks forced running increased hippocampal proliferation and BDNF protein in one-month old rats [7]. The hippocampal proliferation and BDNF protein were also enhanced in middle age mice by 5 weeks treadmill exercise [8]. In our study, voluntary running increased BDNF mRNA and was accompanied by hippocampal proliferation. However, a previous study reported that short-term treadmill exercise enhanced proliferation in the DG but did not increase BDNF protein in 2-month old Wistar rats [10]. Another study also reported that 1-month running did not enhance hippocampal proliferation and BDNF protein [11]. These discrepancies may be associated with exercise intervention duration. It is likely that the effect of exercise-induced hippocampal proliferation is higher if the duration of voluntary exercise is longer. Hippocampal proliferation is also likely associated with BDNF expression. Two possible subcellular cascades may underlie these changes. First, BDNF may activate the TrkB receptor, which in turn increases CREB phosphorylation via the MAPK pathway, and promotes hippocampal neuronal proliferation. Second, phosphorylation of the TrkB receptor may activate PI3K, which then phosphorylates Akt to promote hippocampal proliferation [23]. Thus, BDNF may be a mediator for exercise-induced neuronal proliferation.

The function of IGF-1 in regulating adult hippocampal proliferation has been demonstrated in previous studies [24, 25]. In the present study, we found that voluntary exercise increased the expression of IGF-1 in the hippocampus, which was in line with other findings [26, 27]. One explanation for this finding could be that exercise enhances IGF-1 production in the liver, and this hepatic IGF-1 might be transported to the brain via the bloodstream, resulting in the increased IGF-1 expression in the hippocampus [13, 28]. Further, we observed that voluntary running not only increased hippocampal IGF-1 mRNA, but also promoted hippocampal proliferation. Therefore, the increase IGF-1 correlated with hippocampal proliferation. IGF-1 activated IGF-1 receptors, which in turn activate the MAPK and PI3K-Akt signal pathways to induce hippocampal proliferation [6, 29]. Thus, the enhancement of IGF-1 following exercise may also play a role in exercise-induced hippocampal proliferation. Consistent with this, the results from a previous study suggest that exercise increases IGF-1 expression in the brain, which enhances BDNF and promotes hippocampal proliferation [30]. 


\section{Conclusion}

Voluntary running improved hippocampal proliferation in mice, which was associated with the upregulation of NGF, BDNF, and IGF-1. However, more research is needed to determine which factor is more important in exercise-induced proliferation and to elucidate the interaction between these factors.

\section{Acknowledgments}

This research was supported by grants from the National Natural Science Foundation of China (Grant No. 31571225, 31650006).

\section{References}

[1] Kamada M, Li RY, Hashimoto M, Kakuda M, Okada H, Koyanagi $\mathrm{Y}$ et al.. Intrinsic and spontaneous neurogenesis in the postnatal slice culture of rat hippocampus. Eur J Neurosci 2004; 20: 2499-2508.

[2] Zhao C, Deng W, Gage FH. Mechanisms and functional implications of adult neurogenesis. Cell 2008; 132: 645-660.

[3] Deng W, Aimone JB, Gage FH. New neurons and new memories: how does adult hippocampal neurogenesis affect learning and memory? Nat Rev Neurosci 2010; 11: 339-350.

[4] Birch AM, Kelly AM. Chronic intracerebroventricular infusion of nerve growth factor improves recognition memory in the rat. Neuropharmacology 2013; 75: 255-261.

[5] Choi SH, Li Y, Parada LF, Sisodia SS. Regulation of hippocampal progenitor cell survival, proliferation and dendritic development by BDNF. Mol Neurodegener 2009; 4: 52.

[6] Aberg MA, Aberg ND, Palmer TD, Alborn AM, Carlsson-Skwirut $\mathrm{C}$, Bang $\mathrm{P}$ et al.. IGF-I has a direct proliferative effect in adult hippocampal progenitor cells. Mol Cell Neurosci 2003; 24: 23-40.

[7] Ji JF, Ji SJ, Sun R, Li K, Zhang Y, Zhang LY et al.. Forced running exercise attenuates hippocampal neurogenesis impairment and the neurocognitive deficits induced by whole-brain irradiation via the BDNF-mediated pathway. Biochem Biophys Res Commun 2014; 443: 646-651.

[8] Wu CW, Chang YT, Yu L, Chen HI, Jen CJ, Wu SY et al. Exercise enhances the proliferation of neural stem cells and neurite growth and survival of neuronal progenitor cells in dentate gyrus of middle-aged mice. J Appl Physiol (1985) 2008; 105: 1585-1594.

[9] Bechara RG, Kelly AM. Exercise improves object recognition memory and induces BDNF expression and cell proliferation in cognitively enriched rats. Behav Brain Res 2013; 245: 96-100.

[10] Ferreira AF, Real CC, Rodrigues AC, Alves AS, Britto LR. Short-term, moderate exercise is capable of inducing structural, BDNF-independent hippocampal plasticity. Brain Res 2011; 1425: $111-122$.

[11] Marlatt MW, Potter MC, Lucassen PJ, van Praag H. Running throughout middle-age improves memory function, hippocampal neurogenesis, and BDNF levels in female C57BL/6J mice. Dev Neurobiol 2012; 72: 943-952.

[12] Nakajima S, Ohsawa I, Ohta S, Ohno M, Mikami T. Regular voluntary exercise cures stress-induced impairment of cognitive function and cell proliferation accompanied by increases in cerebral IGF-1 and GST activity in mice. Behav Brain Res 2010; 211: 178-184.

[13] Trejo JL, Carro E, Torres-Aleman I. Circulating insulin-like growth factor I mediates exercise-induced increases in the number of new neurons in the adult hippocampus. $J$ Neurosci 2001; 21: 1628-1634.

[14] Neeper SA, Gomez-Pinilla F, Choi J, Cotman CW. Physical activity increases mRNA for brain-derived neurotrophic factor and nerve growth factor in rat brain. Brain Res 1996; 726: 49-56.

[15] Yuede CM, Zimmerman SD, Dong H, Kling MJ, Bero AW, Holtzman DM et al.. Effects of voluntary and forced exercise on plaque deposition, hippocampal volume, and behavior in the Tg2576 mouse model of Alzheimer's disease. Neurobiol Dis 2009; 35: 426-432.

[16] Allen DL, Harrison BC, Maass A, Bell ML, Byrnes WC, Leinwand LA. Cardiac and skeletal muscle adaptations to voluntary wheel running in the mouse. $J$ Appl Physiol (1985) 2001; 90: 1900-1908.

[17] van Praag H, Kempermann G, Gage FH. Running increases cell proliferation and neurogenesis in the adult mouse dentate gyrus Nat Neurosci 1999; 2: 266-270.

[18] van Praag H, Shubert T, Zhao C, Gage FH. Exercise enhances learning and hippocampal neurogenesis in aged mice. $J$ Neurosci 2005; 25: 8680-8685.

[19] Lou SJ, Liu JY, Chang H, Chen PJ. Hippocampal neurogenesis and gene expression depend on exercise intensity in juvenile rats. Brain Res 2008; 1210: 48-55.

[20] O'Callaghan RM, Griffin EW, Kelly AM. Long-term treadmill exposure protects against age-related neurodegenerative change in the rat hippocampus. Hippocampus 2009; 19: 1019-1029.

[21] Kim HG, Oh MS. Memory-enhancing effect of Mori Fructus via induction of nerve growth factor. Br J Nutr 2013; 110: 86-94.

[22] Scharfman H, Goodman J, Macleod A, Phani S, Antonelli C, Croll S. Increased neurogenesis and the ectopic granule cells after intrahippocampal BDNF infusion in adult rats. Exp Neurol 2005; 192: 348-356.

[23] Islam O, Loo TX, Heese K. Brain-derived neurotrophic factor (BDNF) has proliferative effects on neural stem cells through the truncated TRK-B receptor, MAP kinase, AKT, and STAT- 3 signaling pathways. Curr Neurovasc Res 2009; 6: 42-53.

[24] Lichtenwalner RJ, Forbes ME, Bennett SA, Lynch CD, Sonntag WE, Riddle DR. Intracerebroventricular infusion of insulin-like growth factor-I ameliorates the age-related decline in hippocampal neurogenesis. Neuroscience 2001; 107: 603-613.

[25] Carro E, Torres-Aleman I. Serum insulin-like growth factor I in brain function. Keio J Med 2006; 55: 59-63. 
[26] Ding Q, Vaynman S, Akhavan M, Ying Z, Gomez-Pinilla F. Insulin-like growth factor I interfaces with brain-derived neurotrophic factor-mediated synaptic plasticity to modulate aspects of exercise-induced cognitive function. Neuroscience 2006; 140: 823-833.

[27] Cetinkaya C, Sisman AR, Kiray M, Camsari UM, Gencoglu C, Baykara B et al.. Positive effects of aerobic exercise on learning and memory functioning, which correlate with hippocampal IGF-1 increase in adolescent rats. Neurosci Lett 2013; 549: 177-181.

[28] Carro E, Trejo JL, Busiguina S, Torres-Aleman I. Circulating insulin-like growth factor I mediates the protective effects of physical exercise against brain insults of different etiology and anatomy. J Neurosci 2001; 21: 5678-5684.

[29] Choi YS, Cho HY, Hoyt KR, Naegele JR, Obrietan K. IGF-1 receptor-mediated ERK/MAPK signaling couples status epilepticus to progenitor cell proliferation in the subgranular layer of the dentate gyrus. Glia 2008; 56: 791-800.

[30] Carro E, Nunez A, Busiguina S, Torres-Aleman I. Circulating insulin-like growth factor I mediates effects of exercise on the brain. J Neurosci 2000; 20: 2926-2933. 